\title{
Concordancia entre el diagnóstico clínico y paraclínico de las cervico-vaginitis más frecuentes
}

\author{
Javier Ardila Montealegre* y Cols.; Jorge Gómez** y Cols.; Edith Angel M.*** y Cols.
}

\begin{abstract}
RESUMEN: Se realizó un estudio multicéntrico (Hospital Universitario de San Ignacio, Hospital San José e Instituto Materno Infantil de Bogotá), seleccionando secuencialmente 301 pacientes de la consulta general de ginecología con o sin sintomatología de cervico-vaginitis que cumplieran los criterios de inclusión y de exclusión. El objetivo general del estudio fue establecer la concordancia entre el diagnóstico clínico de la secreción vaginal y el diagnóstico hecho mediante los exámenes para-clínicos considerados como estándar de oro. Dentro de los objetivos específicos se consideraron establecer las cervico vaginitis tanto sintomáticas como asintomáticas, establecer la sensibilidad y especificidad del examen clínico frente al método diagnóstico para cada germen en particular y establecer la frecuencia con que las cervicovaginitis se presentan en nuestro medio. A cada paciente se le practicó inicialmente examen con especuloscopia realizando el diagnóstico clínico correspondiente a los hallazgos encontrados, y posteriormente se le tomó muestra de flujo específicamente para Cultivo de Gardnerella vaginalis, Trichomona vaginalis, Candida albicans, Neisseria gonorreae y prueba de ELISA para Clamydia trachomatis. La prevalencia de cervico-vaginitis sintomática y asintomática en nuestras pacientes fue de $67.4 \%$ y $32.2 \%$ respectivamente. La presencia de los gérmenes específicos en nuestro medio fue: Gardnerella vaginalis $23.7 \%$, Clamydia trachomatis $14.3 \%$, Candida albicans $8.4 \%$. Trichomona vaginalis $1.7 \%$ y Gonococo $1 \%$. La sensibilidad y la especificidad de los diagnósticos clínicos fue respectivamente $61.5 \%$ y $66.4 \%$ para Candida albicans; $76.9 \%$ y $79.3 \%$ para Gardnerella vaginalis; $100 \%$ y $87,9 \%$ para trichomona vaginalis; $0 \%$ y $98.6 \%$ para infección gonocóccica y $36.36 \%$ y $95.71 \%$ para Clamydia trachomatis.
\end{abstract}

PALABRAS CLAVES: Cervico-vaginitis, sintomática, asintomática, diagnóstico clínico y de laboratorio, sensibilidad, especificidad, Gardnerella vaginalis, Candida albicans, Neisseria gonorrhoeae, Clamydia trachomatis.

SUMMARY: The results of a multicenter study which included the Hospital Universitario San Ignacio, Hospital San José and the Instituto Materno Infantil of Bogotá are presented. Three hundred and one consecutive women, with or without symptoms who fulfilled the inclusion criteria were studied. The general objective of the study was to establish the correlation between the clinical diagnosis of the vaginal secretion and the diagnosis by gold standard laboratory methods. The specific objectives were to establish the prevalence of symptomatic and asymptomatic cervicovaginitis; to determine the sensitivity and specificity of the clinical examination in front to the laboratory diagnosis for each particular germ; and to establish the frequency of cervicovaginitis in our patients. Initially an examination with speculum and clinical diagnosis were done and them a culture for Gardnerella vaginalis, Trichomona vaginalis, Candida albicans, Neisseria gonorreae and an ELISA test for Clamydia trachomatis were practiced. The prevalences of the symptomatic and asymptomatic cervicovaginitis in our patients were $67.4 \%$ y $32.2 \%$ respectively. The frequency of the specific germs inour patients were: Gardnerella vaginalis $23.7 \%$, Clamydia trachomatis $14.3 \%$, Candida albicans $8.4 \%$, Trichomona vaginalis $1.7 \%$ and Gonococo $1 \%$. The sensivity and specificity of the clinical diagnosis were respectively $61.5 \%$ and $66.4 \%$ for Candida albicans; $76.9 \%$ and $79.3 \%$ for Gardnerella vaginalis; $100 \%$ and $87,9 \%$ for Trichomona vaginalis; $0 \%$ and $98.6 \%$ for Gonococal infection and $36.36 \%$ and $95.71 \%$ for Clamydia trachomatis.

KEY WORDS: Cervico-vaginitis, symptomatic, asymptomatic, clinical and laboratory diagnosis, sensitivity, specificity, Gardnerella vaginalis, Trichomona vaginalis, Candida albicans, Neisseria gonorrhoeae, Clamydia trachomatis.

El flujo vaginal es el síntoma más frecuente por el cual las mujeres consultan tanto al ginecólogo como al médico general. Por otro lado, en algunas mujeres asintomáticas que no consultan puede estar presente y corresponder a etiologías específicas como las conside-

\footnotetext{
- Estudio Interinstitucional.

* Hospital San Ignacio.

** Hospital San José

*** Instituto Materno Infantil.
}

radas en el actual estudio. Estas razones son de importancia para determinar de una manera adecuada el germen causal y actuar en consecuencia con un tratamiento igualmente adecuado. Sin embargo, la frecuencia con que se presenta esta patología ha hecho que el diagnóstico tome connotaciones diagnósticas puramente clínicas y que en muy pocas ocasiones se recurra a los métodos considerados como estándar de oro (1). Esta práctica es común y puede tener repercusiones no sólo desde el punto de vista de tratamiento adecuado sino también de sobre-formulación de antibióticos tanto sistémicos como locales. Por otro lado, no todos los flujos vaginales 
indican la presencia de un agente infeccioso, por lo que los tratamientos administrados por la misma paciente o por el médico sin identificar la causa del flujo, pueden empeorar la condición de la paciente (2).

El tracto genital femenino normalmente tiene una flora microbiana muy variada que en condiciones patológicas y cuando se reúnen ciertas condiciones ideales para su desarrollo puede hacerse invasora para producir cérvico-vaginitis y ser la puerta de entrada a las enfermedades pélvicas inflamatorias, con toda la gama de secuelas que conocemos y que se centran fundamentalmente en el futuro reproductivo de la paciente.

La prevalencia de la tricomoniasis varía ampliamente dependiendo de los grupos poblacionales en los cuales se estudia. En las pacientes asintomáticas en los Estados Unidos se identifica el $5 \%$ con la enfermedad, mientras que en el grupo de las prostitutas se encuentra en el 50$75 \%(3-4)$.

La prevalencia de este tipo de infecciones en nuestro medio depende de varios factores tales como promiscuidad, aumento de enfermedades de transmisión sexual, la falta de uso de métodos de barrera, el uso indiscriminado de tratamientos (5), lo cual hace que en la última década haya cambiado sustancialmente y por tanto ha aumentado la posibilidad de que el diagnóstico clínico tenga menos probabilidades de ser acertado.

Se han encontrado datos de importancia en cuanto al endocérvix como reservorio de microorganismos tales como Gardnerella, Trichomona y Gonococo, los cuales se transmiten fundamentalmente por contacto sexual (8). De tal manera que es poco práctico intentar establecer diagnósticos independientes de cervicitis y cervicovaginitis porque coexisten en más de la mitad de las veces, con importancia clínica dada por potencial diseminación a sus compañeros sexuales y en el caso de las mujeres gestantes generando patologías como parto pretérmino, ruptura prematura de membranas e infecciones fetales o del recién nacido (10).

\section{Materiales y métodos}

El protocolo comprende tres fases:

1. Estandarización; asegurando igual desempeño en las tres instituciones en cuanto a:

- Toma de la muestra

- Recolección de la información

- Procesamiento de la muestra en el laboratorio (Criterios bacteriológicos y técnicos)

Para llevar a término esta fase se realizaron múltiples reuniones con los diferentes participantes de cada institución con el ánimo de tratar situaciones específicas en lo que respecta a la parte clínica y de laboratorio, que eventualmente podrían ser causales de divergencia, hasta que se lograra a un acuerdo común con el cual todos los participantes pudieran actuar al unísono.

2. Estudio piloto; asegurando:

Identificación de los errores en el montaje y desarrollo del protocolo tanto en los investigadores como en la concepción logística. Una vez se estandarizan los criterios, se inicia la recolección de la información para poder establecer cuales son las fuentes de posibles dificultades que no hubieran sido consideradas con antelación. Esto se logra mediante reuniones de los participantes en cada uno de los centros durante el proceso de selección de las pacientes, recolección de las muestras y recolección de la información. Esto permitió tener una visión más real de como era el funcionamiento de cada institución en particular para hacer los ajustes necesarios.

3. Desarrollo del protocolo propiamente dicho

Los criterios de inclusión que se tuvieron en cuenta fueron:

1. Toda aquella paciente que asista a consulta ginecológica con o sin sintomatología de cérvico-vaginitis.

2. Edad que oscile entre 16-45 años

3. Consentimiento informado de la paciente para ser incluida en el estudio

Los criterios de exclusión considerados fueron:

1. Coito en las 24 horas previas a la toma de la muestra

2. Ducha vaginal en las 24 horas previas a la toma de la muestra

3. Presencia de menstruación o sangrado genital de cualquier causa en el momento de la toma de la muestra

4. Condiciones de la paciente que impidan el examen ginecológico

5. Enfermedad de base que modifique el micro-ambiente vaginal (Diabetes, Cáncer, Enfermedades autoinmunes).

6. Tratamiento con antibióticos o medicamentos diferentes en cualquiera de sus presentaciones utilizados para infecciones en las dos semanas previas a la toma de la muestra.

La selección de las pacientes se hace de manera secuencial en cada institución y previo consentimiento informado, se realiza el interrogatorio correspondiente de acuerdo con un formulario previamente establecido para tal efecto. El examen se lleva a cabo inicialmente con la inspección de los genitales externos y posteriormente con la especuloscopia sin utilizar aceite o cualquier otro lubricante.

Se obtienen muestras de la secreción vaginal así:

Endocérvix: Con un hisopo estéril para realizar coloración de Gram.

Otro hisopo estéril para cultivo en Thayer Martín Otro hisopo especial para identificación de Clamydia (Kit Clearview Clamydia).

Exocérvix: Con hisopo estéril se toma muestra exocervical y de fondo de saco para realizar la prueba de aminas volátiles con $\mathrm{KOH} 10 \%$.

Segunda muestra con otro hisopo para coloración de Gram

Tercera muestra con hisopo para cultivo de Saboureau, Tricosel y Agar sangre.

Se realiza además lectura en fresco para identificación de Trichomona y Gardnerella y lectura del $\mathrm{KOH}$ para levaduras, pseudomicelios y micelios.

\section{Resultados}

Se incluyeron 301 pacientes

* 100 Hospital San Ignacio

* 100 Hospital San José

* 101 Instituto Materno Infantil 
TABLAS DE CARACTERÍSTICAS GENERALES:

\begin{tabular}{|cccc|}
\hline Edad & Frecuencia (\%) & Paridad & Frecuencia (\%) \\
\hline $15-25$ & 15.9 & 0 & 12.6 \\
$26-35$ & 43.0 & $1-3$ & 62.8 \\
$36-45$ & 41.0 & $>3$ & 24.6 \\
\hline
\end{tabular}

\begin{tabular}{|lc|}
\hline Planificación & Frecuencia (\%) \\
\hline DIU & 20.3 \\
Anov. orales & 11.3 \\
Barrera & 9.6 \\
Pomeroy & 15.9 \\
Ninguno & 41.9 \\
\hline
\end{tabular}

\begin{tabular}{|lc|}
\hline pH Fondo de saco & Frecuencia (\%) \\
$<7$ & 87.6 \\
$\geq 7$ & 12.4 \\
\hline
\end{tabular}

Se observaron los siguientes resultados:

La frecuencia con que las pacientes describen o no la presencia de sintomatología en lo que respecta a evidencia del flujo que es experimentado por ellas como anormal, es:

\begin{tabular}{|lc|}
\hline Cérvico-vaginitis & Prevalencia \\
Sintomática & $67,4 \%$ \\
Asintomática & $32.2 \%$ \\
\hline
\end{tabular}

Esta información adquiere importancia en la medida en que este síntoma es tan común y de larga data que muchas de las pacientes en que se evidencia se habitúan a su presencia, hasta el punto de referirlo como normal y auto-calificarse como "asintomáticas".

En cuanto a la prevalencia de los gérmenes causales, podemos asumir de cierta manera que la frecuencia con que se presentaron dentro de la población incluida en el protocolo, puede ser equivalente a la prevalencia en la población general ya que las instituciones participantes representan sus diferentes estratos socio-económicos, haciendo la aclaración de que un dato más fidedigno de prevalencia hubiese necesitado una población mayor.

En lo que respecta a las características intrínsecas del examen clínico asumido como prueba diagnóstica de práctica común en nuestro medio, con respecto al estándar de oro para cada uno de los gérmenes tenidos en cuenta por el estudio

(Cultivos), tenemos los siguientes hallazgos:

\begin{tabular}{|lcc|}
\hline Diagnóstico clínico & Sensibilidad & Especificidad \\
\hline Candida Albicans & $61,53 \%$ & $66,35 \%$ \\
Gardnerella vaginalis & $76,92 \%$ & $79,26 \%$ \\
Trichomona vaginalis & $100 \%$ & $87,95 \%$ \\
Gonococo & $0 \%$ & $98,6 \%$ \\
Clamydia trachomatis & $36,36 \%$ & $95,71 \%$ \\
\hline
\end{tabular}

Observamos como la capacidad del examen clínico para identificar a las pacientes realmente enfermas como enfermas (Sensibilidad), en términos generales es muy mala, sin embargo, la capacidad para identificar a las pacientes realmente sanas corno sanas (Especificidad) es mejor, aunque es importante recordar que aunque sean bajos los falsos negativos, el precio que pagamos por no detectar la enfermedad puede ser alto, si pensamos en que las consecuencias pueden manifestarse en un pronóstico sombrío en lo que compete al futuro reproductivo de la paciente y a un aumento del contagio de la respectiva enfermedad mediante el contacto sexual hetero y/o homosexual. Esto implica, que lo que necesitamos para el diagnóstico es una prueba altamente sensible que nos permita actuar en consecuencia y administrar un tratamiento oportuno y eficaz.

Los gérmenes estudiados se presentaron en el siguiente orden de frecuencia:

\begin{tabular}{|lc|}
\hline Germen causal & Frecuencia \\
\hline Gardnerella vaginalis & $23,7 \%$ \\
Clamydia trachomatis & $14,3 \%$ \\
Candida albicans & $8,4 \%$ \\
Trichomona vaginalis & $1,7 \%$ \\
Gonococo & $1,0 \%$ \\
\hline
\end{tabular}

Es importante enfatizar el giro de la frecuencia en la última década con respecto a los gérmenes estudiados. Hace aproximadamente 15 años las principales etiologías estaban dadas por Trichomona y Gonococo, sin embargo, actualmente éstos ocupan los últimos lugares en frecuencia en nuestro estudio. Mientras, la Gardnerella vaginalis y la Clamydia trachomatis ocupan respectivamente los dos primeros lugares en frecuencia, lo cual es importante si tenemos en cuenta que la vaginosis es una de las principales causas de amenaza de trabajo de parto pretérmino y de endometritis y por otro lado la Clamydia representa un germen cuya prevalencia en nuestro medio no ha sido estudiada hasta el momento y en Estados Unidos genera un gran porcentaje de enfermedades pélvicas inflamatorias en pacientes jóvenes. Este hallazgo implica que nuestros esfuerzos deben enfilarse al diagnóstico racional de estos gérmenes.

Las combinaciones más frecuentemente encontradas corresponden a:

\begin{tabular}{|c|c|}
\hline Gardnerella + Clamydia & $\begin{array}{l}7 \text { pacientes }(2.3 \% \text { de la } \\
\text { población total). }\end{array}$ \\
\hline Gardnerella + Candida & $\begin{array}{l}4 \text { pacientes }(1.3 \% \text { de la } \\
\text { población total). }\end{array}$ \\
\hline Candida + Clamydia & $\begin{array}{l}2 \text { pacientes }(0.7 \% \text { de la } \\
\text { población total }) .\end{array}$ \\
\hline
\end{tabular}

En ninguna paciente se encontró la coexistencia de más de 2 gérmenes diferentes.

En cuanto al diagnóstico de los gérmenes más frecuentes, surge la necesidad de identificar si es necesario recurrir siempre al cultivo. El análisis realizado para dar respuesta a este interrogante es el siguiente:

La vaginosis bacteriana, en la cual están presentes la Gardnerella vaginalis y bacterias anaerobias, puede identificarse en los siguientes criterios clínicos: $\mathrm{pH}$ mayor que 4.5 , prueba de aminas positiva y células guía en la 
coloración de Gram. Desde luego, la Gardnerella puede identificarse en el cultivo. De 71 pacientes con cultivo positivo, 62 tuvieron Células Guía positivas en el Gram y 64 tuvieron Células Guía en el fresco de flujo lo que corresponde al $87 \%$ y $90 \%$ respectivamente de la población con flujo producido por Gardnerella. En lo que respecta a la volatilización de aminas mediante la aplicación de $\mathrm{KOH} 10 \%$ encontramos que fue interpretada como positiva en 46 pacientes de las 71 identificadas por el cultivo. Esta cifra corresponde al $64 \%$ de la población con flujo producido por Gardnerella. Esto indica que no es necesario recurrir en todas las pacientes al cultivo para hacer un diagnóstico racional de vaginosis bacteriana.

La Clamydia trachomatis presenta una muy mala sensibilidad para el diagnóstico clínico por tanto, para su identificación debe recurrirse a los métodos inmunológicos (Elisa) o al cultivo en pacientes con o sin factores de riesgo (promiscuidad) o a quienes se les encuentra flujos específicos por otros gérmenes.

Para el diagnóstico de infección por Candida, podemos utilizar el Gram para la identificación de Levaduras puesto que el $96 \%$ de los cultivos positivos para Candida (25) tuvieron además levaduras positivas en la coloración de Gram. El diagnóstico clínico para Candida alcanza una sensibilidad del $61,53 \%$, por ende este diagnóstico no es confiable y debe ser reemplazado por la coloración de Gram.

El Gonococo tiene una prevalencia muy baja en el estudio y probablemente debemos buscarlo teniendo en cuenta las características y factores asociados de cada paciente, centrando el diagnóstico en el cultivo tomado del endocérvix.

La Trichomona a pesar de su baja frecuencia de presentación, guarda una estrecha relación entre el diagnóstico mediante su hallazgo en el fresco de flujo y el cultivo, por tanto no es necesario recurrir a este último para tener un diagnóstico claro de infección cérvicovaginal por este germen.

El método de planificación y el porcentaje con que se presentó la infección por los dos gérmenes más frecuentes fue:

\begin{tabular}{|lccr|}
\hline Cultivo & DIU & Barrera & An. Orales \\
\hline Gardnerella (71) & $23(32.39 \%)$ & $04(5.6 \%)$ & $08(11.2 \%)$ \\
Clamydia (42) & $08(19.04 \%)$ & $04(9.5 \%)$ & $08(19.0 \%)$ \\
\hline
\end{tabular}

\section{Conclusiones}

Los gérmenes estudiados tuvieron el siguiente orden de frecuencia:

Gardnerella, Clamydia trachomatis, Candida albicans, Trichomona vaginalis y por último el gonococo.

Las combinaciones más frecuentes fueron:

Gardnerella con Clamydia, Gardnerella con Candida y Candida con Clamydia.

Este hallazgo hace tener presente que el flujo identificado como específico para cualquiera de estos gérmenes, al cual se le da tratamiento y no mejora, muy probablemente está siendo perpetuado por otro germen que no ha sido identificado.
El cultivo a pesar de ser considerado el estándar de oro, no es necesario en la identificación rutinaria de todos los gérmenes, puesto que hay para-clínicos más baratos, más accequibles en todos los niveles de atención y más fáciles de realizar por lo que sus resultados pueden ser obtenidos más rápidamente.

El diagnóstico clínico etiológico de la cérvicovaginitis muestra una pobre correlación con el diagnóstico por cultivo, por tanto nuestros esfuerzos deben enfilarse a la clara identificación del germen para dar un tratamiento adecuado.

El diagnóstico de Vaginosis bacteriana puede realizarse de manera racional con el hallazgo de células Guía en la coloración de Gram y en el examen en fresco. El cultivo se puede tener como el método diagnóstico para casos particulares que así lo requieran. En el $90 \%$ de las pacientes es suficiente con el Gram y el examen en fresco.

La Clamydia siempre requiere del ELISA para su diagnóstico y no tiene características clínicas que la hagan sospechar, de tal manera que debe solicitarse cuando se sospecha por antecedentes de la historia clínica o a criterio del médico tratante, ya que se encontró de manera indiscriminada tanto en pacientes promiscuas como en pacientes con un solo compañero sexual.

El Gonococo, tuvo una prevalencia muy baja y los hallazgos indican que su diagnóstico siempre debe realizarse con el cultivo endocervical específico.

El diagnóstico de la Trichomona puede hacerse con el examen en fresco del flujo vaginai, donde se evidencia el germen. Por tanto podemos obviar el estándar de oro (cultivo) y continuar con un adecuado método diagnóstico como mostró ser el fresco de flujo.

La Candida puede diagnosticarse de manera confiable con el Gram para la identificación de levaduras. Por lo tanto el cultivo puede ser solicitado únicamente en casos muy especiales en que el diagnóstico sea particularmente difícil.

\section{Discusión}

Los profesionales de la salud que se encuentran enfrentados a este tipo de patologías, podrán argumentar que un considerable número de pacientes que son tratadas de acuerdo a la impresión clínica que se haga del flujo, tienen una resolución de su cuadro; sin embargo, teniendo en cuenta que la baja sensibilidad del examen clínico frente a la idoneidad y simplicidad de los métodos aquí descritos, hace casi mandatorio tener más elementos de juicio para ofrecer tratamientos más efectivos, máxime cuando los datos pueden ser extrapolados a pacientes en estado de embarazo, donde las consecuencias de un manejo inadecuado pueden llegar a cuadros clínicos de mayor envergadura como el parto pretérmino, corioamnionitis y endometritis post-parto.

\section{Agradecimientos}

Extendemos nuestro sincero reconocimiento al apoyo incondicionado de laboratorios Specia, con el cual fue posible llevar a feliz término la realización de este protocolo de investigación. 


\section{BIBLIOGRAFIA}

1. Díaz Federico, Vásquez María Helena, Escobar Sara y Col. Vaginitis por Gardnerella vaginalis en un servicio médico universitario. Rev. Colombiana Obst. Ginecol. 1980; 31(6): 385-394.

2. Soihet, Samoel. El flujo vaginal en la consulta ginecológica. The vaginal discharge in the gynecological consultation. Ginecol \& Obstet; 1986; 30(2): 56-60.

3. Thomason JL., Gelbart SM. Trichomonas vaginalis. Obstet. Gynecol. 1989; 74: 536-541.

4. Rein MF., Muller M. Trichomonas vaginalis and trichomoniasis. Sexually Transmitted Diseases. New York: McGraw Hill; 1989; 481. 492.

5. Lossick JG. Epidemiology of urogenital trichomoniasis. In Honinberg BM, de Trichomonads parasitic en humans. New York: 1989; 311-323.

6. Heredia Reinaldo, Agudelo Clara, Castañeda Elizabeth. Prevalencia de los agentes etiológicos de la vaginitis y la cervicitis en pacientes de consulta ginecológica general. Acta médica Colomb. Marzo-Abril $1990 ; 15(2)$ : 92-99.

7. Tovar Gabriel, Sánchez Enrique. Tratamiento de la vaginitis: Bogotá, CAFAM, Sep. 1988; 665.
8. Segovia Sylvia, Gómez Orietta, Fernández Alejandra y Col. Estudio microbiológico de vaginitis y cervicitis. Rev. Chil. Obstet. Ginecol. 1987; 52(3): 165-177.

9. López Rubén, Ruiz D., Robledo My Col. Significación patogénica de Candida en pacientes con vaginitis. Ginecol. Obstet. Méx. 1982; 50(302): 145-148.

10. Orozco Guillermo, Ospina César, Dilimonto Helena y Col. Guías de manejo clínico: Infecciones cérvico-vaginales Trib. Méd. (Bogotá); 1990.10 82(1): 13-20.

11. Garrocho C., Adame L., Ruiz MP y Col. Candidiasis y trichomoniasis vaginal. Pueden diagnosticarse clínicamente con seguridad? Ginecol. Obstet. Méx. 1983; 51(316): 199-203.

12. Onetto S., Vargas L. Diagnóstico de Gardnerella vaginalis en población no seleccionada con o sin vaginosis bacteriana. Rev. Chil. Tecnol. Med. 1985; 8(2): 347-354.

13. Guerreiro HM., Barbosa H., Conceicao F. y Col. Flora vaginal e correlacao con aspectos citológicos. Rev. Saúde publica. 1986; 20(6): 415-420. 